\title{
WHEN ARE SIMPLE FLASHES AND FLOATERS OCULAR EMERGENCIES?
}

\author{
JEREMY P. DIAMOND \\ Bristol
}

\begin{abstract}
SUMMARY
One hundred and seventy patients presented to the Bristol Eye Hospital casualty department with flashes and/or floaters during a six month prospective study. A sight-threatening condition was found in 41 patients (24.1\%), the most important of which was a retinal break (16.5\%).

Although there were no specific symptoms which could be correlated to an increased incidence of retinal breaks, those patients who complained of isolated uniocular floaters had an insignificant incidence of breakage, when compared to asymptomatic fellow eyes.

Thus while the majority of patients with flashes and floaters do merit an urgent ophthalmological opinion, those who complain of a single, isolated floater can safely be reviewed as routine outpatients.
\end{abstract}

Flashes and floaters may cause patients considerable alarm, whilst general practitioners and ophthalmic opticians recognise them to be harbingers of vitreo-retinal disease. Consequently these patients frequently attend ophthalmic casualty departments, ${ }^{1}$ where considerable resources are required to exclude serious underlying pathology.

Previous studies undertaken to demonstrate the incidence of ocular disease have mostly originated from American secondary or tertiary referral centres. ${ }^{2-5}$ These statistics may not accurately reflect the incidence of significant causation in a British casualty department, where patients are usually primary referrals (and where the service is free).

This prospective study was designed to demonstrate the incidence of sight-threatening lesions in a United Kingdom casualty-based population. Clarification of risk factors for treatable disease will help identify those patients who justify emergency examination. Conversely, determination of features associated with a low incidence of significant disease would allow those patients to be reviewed non-urgently, thereby reserving the acute service for those who most require it.

Correspondence to: Mr. J. P. Diamond, FRCS, FCOphth, Bristol Eye Hospital, Lower Maudlin Street, Bristol BS1 2SX

\section{PATIENTS AND METHODS}

The Bristol Eye Hospital casualty department provides a 24-hour, 365 day emergency service for a population of approximately one million. In 1989 there were 12,000 new patient attendances.

During the six-month period February-July 1989 most new patients were seen by the author. Only those presenting with floaters and/or flashes in isolation were included in the study.

Patients who described a subjective drop in visual acuity or a visual field defect were specifically excluded, because these symptoms strongly suggest significant vitreo-retinal disease and such patients always justify emergency review.

Those entered in the study were categorised by age, sex, symptoms and duration, past ocular history and factors predisposing to retinal detachment. After pupil dilation, both eyes were examined using the indirect ophthalmoscope followed by slit lamp biomicroscopy with Goldmann 3-mirror contact lens.

Patients were categorised by diagnosis as defined on presentation. If localised vitreous haemorrhage precluded full fundal examination they were documented as "vitreous haemorrhage cause unknown' and referred for outpatient review. Some later prove to have significant underlying disease.

Posterior Vitreous Detachment (PVD) was diagnosed by identifying the site of detachment from the optic disc, or by recognising the posterior vitreous face within the mid-vitreous cavity.

The majority of retinal breaks identified were 'U-shaped' tears. All those documented were considered likely to result in retinal detachment if denied prophylactic treatment.

During analysis, diagnoses were classified either as 'benign vitreo-retinal disease'-including patients with no discernible aetiology, migraine or with a so-called 'safe' PVD (defined as an isolated PVD, one associated with retinoschisis or with thin vitreous haemorrhage), or as 'potentially sight-threatening disease'-embracing retinal breaks and detachments, symptomatic lattice degeneration, localised dense vitreous haemorrhage, proliferative diabetic retinopathy and posterior uveitis. 
Table I. Diagnosis in 147 patients with unilateral symptoms

\begin{tabular}{|c|c|c|c|c|c|c|}
\hline Diagnosis & Males & $\%$ & Females & $\%$ & Total & $\%$ \\
\hline Isolated PVD & 16 & 34.0 & 50 & 50.0 & 66 & 44.9 \\
\hline Retinal break & 11 & 23.4 & 15 & 15.0 & 26 & 17.7 \\
\hline Migraine & - & - & 2 & 2.0 & 2 & 1.4 \\
\hline PVD, with retinoschisis & - & - & 3 & 3.0 & 3 & 2.0 \\
\hline PVD, with thin vitreous haemorrhage & 2 & 4.2 & 1 & 1.0 & 3 & 2.0 \\
\hline PVD, with lattice degeneration & 1 & 2.1 & 1 & 1.0 & 2 & 1.4 \\
\hline Diabetic proliferative retinopathy & 1 & 2.1 & 1 & 1.0 & 2 & 1.4 \\
\hline Retinal detachment & - & - & 1 & 1.0 & 1 & 0.7 \\
\hline Posterior Uveitis & - & - & 1 & 1.0 & 1 & 0.7 \\
\hline Normal & 14 & 29.8 & 21 & 21.0 & 35 & 23.8 \\
\hline Total & 47 & 100 & 100 & 100 & 147 & 100 \\
\hline
\end{tabular}

\section{RESULTS}

A total of 170 patients were studied. $111(65.3 \%)$ females and $59(34.7 \%)$ males. Mean age was 60.5 years (range $15-89$ years). A total 336 eyes were examined, of which 193 were symptomatic and 143 were asymptomatic fellow eyes. Twenty three had bilateral symptoms.

Table I gives the diagnosis for patients with unilateral symptoms, while Table II shows the most significant diagnosis made in patients with bilateral symptoms.

Forty nine per cent of those with monocular symptoms had a 'safe' PVD, whilst $23.8 \%$ had no discernible aetiology. Combining these figures were those for migraine $(1.4 \%)$, gives a $74.1 \%$ incidence of benign vitreo-retinal disease. These patients were reassured and discharged from follow-up.

The remaining $25.9 \%$ had potentially sight-threatening disease of which the largest sub-group were those with retinal breaks $(17.7 \%)$.

Only $13.0 \%$ of patients presenting with bilateral symptoms had potentially sight-threatening disease (retinal breaks and localised dense vitreous haemorrhage), while $87.0 \%$ had benign vitreo-retinal disease.

Table III presents clinical data relating to the 170 patients. Forty five patients (26.5\%) had a history predisposing to retinal detachment.

Figure 1 correlates age distribution of all patients alongside those with retinal breaks, whilst Figure 2 illustrates the duration of symptoms within these groups. Table IV relates to patients with monocular symptoms and presents data pertaining to the relationship between subjective number of floaters, presence of flashes and the incidence of breaks.

There were two breaks found in 143 asymptomatic fellow eyes $(1.4 \%)$.

\section{DISCUSSION}

Foster Moore first described photopsia in 1935 ('Moores'

Table II. Major diagnosis in 23 patients with bilateral symptoms

\begin{tabular}{lcc}
\hline Diagnosis & No. patients & $\%$ \\
\hline 'Normal' & 11 & 47.8 \\
PVD & 7 & 30.4 \\
Migraine & 2 & 8.7 \\
Retinal breaks & 2 & 8.7 \\
Vitreous haemorrhage cause unknown & 1 & 4.3 \\
Total & 23 & 100 \\
\hline
\end{tabular}

lightning streaks'), drawing attention to their association with muscae volitantes. ${ }^{6}$ Initially considered benign, their association with retinal disease was established later.

Although the origin of floaters is well understood, the source of flashes is more mysterious. The assumption that they arise as a consequence of direct retinal stimulation during vitreous separation is at variance with their almost invariable referral to the temporal field. ${ }^{3,6}$

In this study, one in four patients $(24.1 \%)$ harboured potentially sight-threatening disease.

Of the $75.9 \%$ with a less significant aetiology, a 'safe' PVD was the most frequent cause $(46.5 \%)$-directly comparable to the $43-83 \%$ incidence reported in previous studies. ${ }^{2.5} 27.1 \%$ of all patients had no obvious abnormality. Some of these patients may have been aware of syneretic vitreous degenerative changes.

The overall $16.5 \%$ incidence of retinal breaks correlates with the $10-34 \%$ incidence estimated in previous studies. $2.4,5.7-9$

Surprisingly, only one patient $(0.6 \%)$ was found to have a retinal detachment, suggesting that most such patients had noticed a subjective drop in acuity or a field defect before presentation and were thus excluded from this study.

Untreated, $33-46 \%$ of symptomatic breaks progress to retinal detachment. ${ }^{10} \mathrm{~A}$ means of distinguishing these individuals from those with benign symptoms would reduce referral rates and allow prompt treatment for those who require it.

Table III. Clinical data and relevant past history relating to all patients with correlation to the incidence of retinal breaks

\begin{tabular}{lccc}
\hline Clinical data & $\begin{array}{c}\text { No. of } \\
\text { patients }\end{array}$ & $\begin{array}{c}\text { No. of } \\
\text { breaks }\end{array}$ & $\%$ \\
\hline Male & 59 & 11 & 18.6 \\
Female & 111 & 17 & 15.3 \\
Predisposing history & & & \\
>5 d Myopia & 10 & 1 & 10.0 \\
FHRD* & 7 & 1 & 14.3 \\
Ocular Trauma & 10 & 0 & 0 \\
Previous retinal detachment & 1 & 0 & 0 \\
ECCE*1 & 7 & 0 & 0 \\
ICCE*2 & 4 & 0 & 0 \\
Previous breaks & 5 & 2 & 40.0 \\
Previous vitreous haemorrhage & 1 & 0 & 0 \\
Total & 45 & 4 & 8.9 \\
\hline
\end{tabular}

*Family history of retinal detachment; ${ }^{* 1}$ Extracapsular catract extraction; ${ }^{* 2}$ Intracapsular cataract extraction 


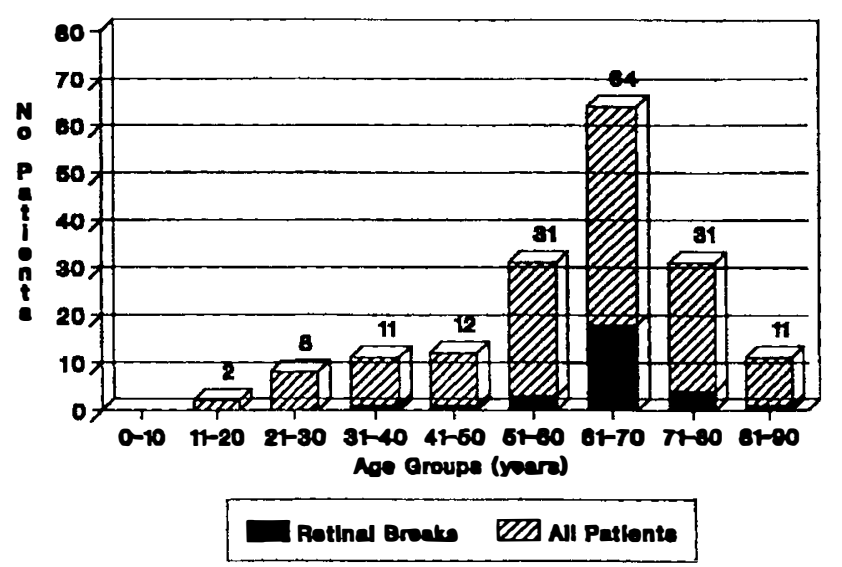

Fig 1. Age of all patients with incidence of retinal breaks.

Boldrey $^{3}$ identified tears in $18.5 \%$ of patients with floaters and flashes. The incidence amongst those with multiple floaters increased to $51.7 \%$. Other high risk groups included those with vitreous pigment or grossly visible vitreous or pre-retinal blood.

In this study there was an increasing incidence of retinal breaks when correlated to length of history (Fig. 2). However, the $29.6 \%$ incidence of retinal breaks seen in patients with a one day history is not significantly higher than the rate thereafter $\left(\chi^{2}\right.$ with Yate's correction $=2.98$, d.f. $=1$, not significant).

Twenty six breaks occurred in 137 patients aged over fifty years $(19.0 \%)$, while only two breaks were found in 33 patients aged 50 years or less $(6.1 \%)$. This increasing incidence of retinal breaks with age is not significant $\left(\chi^{2}\right.$ with Yate's correction $=2.34$, d.f. $=1$, not significant $)$.

Of a total 45 patients $(26.5 \%)$ with a history predisposing to retinal detachment, just four (8.9\%) actually had breaks. Even for those patients with previously treated retinal breaks the incidence of new breaks was not significantly increased above the overall figure (Fishers Exact Probability $=0.06$, not significant).

Although the incidence of retinal breaks in patients with bilateral symptoms $(8.7 \%)$ was considerably lower than that found with unilateral symptoms $(17.7 \%)$, the difference was not significant $\left(\chi^{2}\right.$ with Yate's correction $=0.61$, d.f. $=1$, not significant $)$.

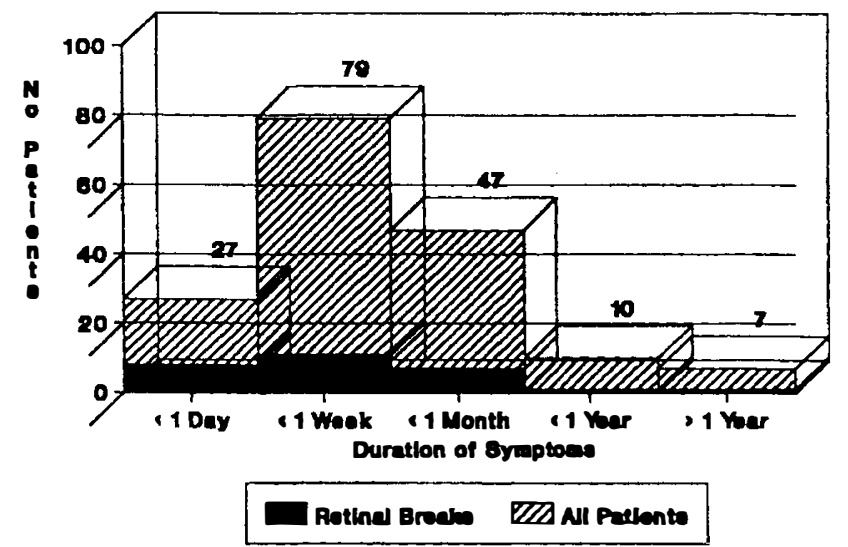

Fig 2. Duration of symptoms of all patients with incidence of retinal breaks.
Table IV. Unilateral symptoms correlated to incidence of retinal breaks

\begin{tabular}{lccc}
\hline Symptoms & No. patients & No. breaks & $\%$ \\
\hline Single floaters & 27 & 1 & 3.7 \\
Multiple floaters & 43 & 8 & 18.6 \\
Flashes alone & 20 & 3 & 15.0 \\
Floaters \& flashes & 57 & 14 & 24.6 \\
Total & 147 & 26 & 17.7 \\
\hline
\end{tabular}

Furthermore, there was no significant difference in incidence of retinal breaks between patients with various types of unilateral symptoms, be they multiple floaters without flashes, flashes without floaters or combined flashes and floaters. $\left(\chi^{2}=1.02\right.$, d.f. $=2$, not significant). However, the numbers studied were small and may be insufficient to expose a clinically significant difference between these groups.

Isolated floaters were generally benign, there being no significant difference in the incidence of breaks when these patients were compared to asymptomatic eyes $\left(\chi^{2}\right.$ with Yate's correction $=0.001$, d.f. $=1$, not significant).

In summary, the most important pathological conditions associated with flashes and/or floaters are retinal breaks, being found in an overall $16.5 \%$ of patients. Although there were no patient sub-groups whose symptoms were associated with an increased incidence of breaks, those who complained of isolated floaters were not at significant risk. Thus, with the exception of this later group, all patients who complain of flashes and/or floaters should be investigated without delay.

I should like to thank Mr. A. Hughes for guidance with the statistical analysis and Mr. R. H. B. Grey for helpful suggestions whilst writing this paper.

\section{REFERENCES}

1. Vernon SA: Analysis of all new cases seen in a busy regional ophthalmic casualty department during 24-week period. J Roy Soc Med 1983, 76: 279-82.

2. Murakami K, Jalkh AE, Alvia MP, Trempe CL, Shepens CL: Vitreous floaters. Ophthalmology 1983, 90: 1271-6.

3. Boldrey EE: Risk of retinal tears in patients with vitreous floaters. Am J Ophthalmol 1983, 96: 783-87.

4. Jaffe NS: Complications of acute posterior vitreous detachment. Arch Ophthalmol 1986, 79: 568-71.

5. Morse PH: Symptomatic floaters as a clue to vitreoretinal disease. Ann Ophthalmol 1975, 7: 865-68.

6. Foster Moore R: Subjective 'lightning streaks'. Br J Ophthalmol 1935, 19: 545-47.

7. Kanski JJ: Complication of acute posterior vitreous detachment. Am J Ophthalmol 1975, 80: 44-6.

8. Linder B: Acute posterior vitreous detachment and its retinal complications. Acta Ophthalmol 1966, 87 (suppl): 1.

9. Tasman WS: Posterior vitreous detachment and peripheral retinal breaks. Trans Am Acad Ophthalmol Otolaryngol 1968, 72: 217-23.

10. Davis MD: Natural history of retinal breaks without detachment. Arch Ophthalmol 1974; 92: 183-194. 